\title{
Metal abundances in the Galactic Center
}

\author{
Francisco Najarro de la Parra \\ Instituto de Estructura de la Materia, CSIC, \\ Serrano 121, 28006 Madrid, Spain \\ D. John Hillier \\ Department of Physics and Astronomy, University of Pittsburgh, \\ 3941 O'Hara Street, Pittsburgh, PA 15260, USA \\ Donald F. Figer \\ Department of Physics 8 Astronomy, University of California, \\ Los Angeles, CA 90095-1562, USA
}

\begin{abstract}
Recent progress in model atmospheres for hot stars is presented. The impact of NLTE wind-blanketed models is shown by comparison with the 'old' standard model. In order to confindently use the IR-spectra of objects at the Galactic Center (with high extinction) to derive their physical properties and trace the metal content, the consistency between results from optical and IR analysis is checked in a calibration study using the $\mathrm{B} 1.5 \mathrm{Ia}^{+}$star HDE 316285 . With the above consistency proved, we started a detail investigation of the Pistol Star. Preliminary results are presented.
\end{abstract}

The influence of line-blanketing is striking (see also Hillier, these Proceedings). A dramatic change in the flux distribution is produced when the blanketing of iron is accounted for with respect to the models with no blanketing, and the effects of UV (blocking) and backwarming are clearly seen. Also the ionization structure of hydrogen throughout the wind (and therefore the line profiles as well) is specially sensitive to line-blanketing.

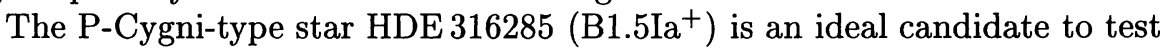
the reliability of the metal abundances determined with the 'new' line-blanketed method as it is one of the few objects which clearly exhibits wind emission metal lines on its spectrum and for which high resolution optical, near-IR and ISOSWs spectra are available (Hillier et al. 1998). Thus, we used this object as 'calibrator' for further metallicity studies of Galactic Center sources based on near-IR observations. The above authors have recently presented a spectroscopic study using the standard 'old' model based on optical and near-IR spectra for $\mathrm{H}, \mathrm{He} \& \mathrm{~N}$. Preliminary results with the 'new' line-blanketed model including $\mathrm{H}, \mathrm{He}, \mathrm{C}, \mathrm{N}, \mathrm{O}, \mathrm{Mg}, \mathrm{Al} \& \mathrm{Fe}$ show that very good agreement with the optical, near-IR and ISO-swS observations is found not only for the $\mathrm{H}$, He I \& N II lines but also for the strong Fe II \& Mg II lines in all wavelength ranges. All lines fitted here are formed in the stellar wind. Our results indicate that the object is considerably evolved and metal rich. The Fe II and Mg II abundances imply roughly twice solar metallicity. 


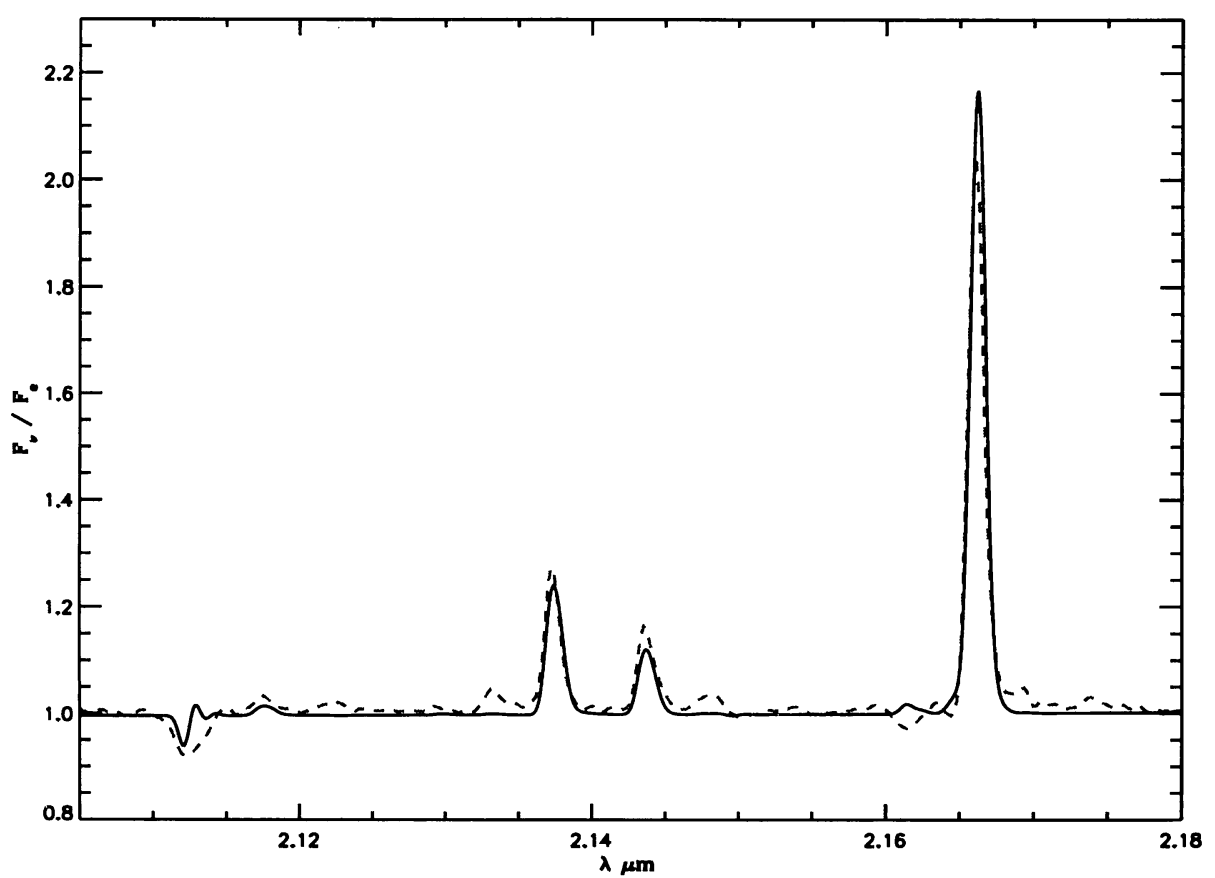

Figure 1. Comparison of the blanketed model (solid) and the observed (grey dashed) $K$-band spectrum of the Pistol Star.

The Pistol Star (Figer et al. 1998) also presents Fe II and Mg II emission lines on its near-IR spectra. Preliminary results of a quantitative spectroscopic investigation with the the 'new' line blanketed method (see Fig. 1) reveal that the $\mathrm{Fe}$ and $\mathrm{Mg}$ content is further enhanced than for HDE $316285(3 \times$ solar metallicity for $\mathrm{Mg}$ and a little bit higher than $3 \times$ solar for $\mathrm{Fe}$ ). These results are in contradiction with those derived by the Ohio group (Sellgren \& Ramirez, priv. comm.) who obtained basically solar metallicity from differential studies of red supergiants in the Galactic Center. Further work in other sources showing metal lines (e.g., IRS $16 \mathrm{NE}$ ) will bring more light into this issue.

Acknowledgments. F. Najarro acknowledges grants of the DGYCIT under PB96-0883 and ESP98-1351.

\section{References}

Figer, D.F., Najarro, F., Morris, M., McLean, I.S., Geballe, T.R., Ghez, A.M., Langer, N. 1998, ApJ 384, 404

Hillier, D.J., Crowther, P.A., Najarro, F., Fullerton, A.W. 1998, A\&A 340, 483 